Marina Silveira Leite

\title{
Kerma no ar com base no índice de exposição para radiografia digital
}


Marina Silveira Leite

\section{Kerma no ar com base no índice de exposição para radiografia digital}

Dissertação apresentada à Faculdade de Filosofia, Ciências e Letras de Ribeirão Preto da Universidade de São Paulo para obtenção do título de Mestre em Ciências. Área de concentração: Física Aplicada à Medicina e Biologia

Orientador: Prof. Dr. Alessandro Martins da Costa

UNIVERSIDADE DE SÃO PAULO

Faculdade de Filosofia, CiÊnCias e Letras de Ribeirão Preto

Ribeirão Preto 
AUTORIZO A REPRODUÇÃO E DIVULGAÇÃO TOTAL OU PARCIAL DESTE TRABALHO, POR QUALQUER MEIO CONVENCIONAL OU ELETRÔNICO, PARA FINS DE ESTUDO E PESQUISA, DESDE QUE CITADA A FONTE.

FICHA CATALOGRÁFICA

Leite, Marina Silveira.

Kerma no ar com base no índice de exposição para radiografia digital. Ribeirão Preto, 2012.

32 p.: il.; $30 \mathrm{~cm}$

Dissertação de Mestrado, apresentada à Faculdade de Filosofia, Ciências e Letras de Ribeirão Preto/USP. Área de concentração: Física Aplicada à Medicina e Biologia.

Orientador: Costa, Alessandro Martins da.

1. Radiodiagnóstico. 2. Dosimetria. 3. Índice de exposição. 4. Radiografia digital. 5. Níveis de referência. 


\section{FOLHA DE APROVAÇÃO}

Marina Silveira Leite

Kerma no ar com base no índice de exposição para radiografia digital

Dissertação apresentada à Faculdade de Filosofia, Ciências e Letras de Ribeirão Preto da Universidade de São Paulo para obtenção do título de Mestre em Ciências. Área de concentração: Física Aplicada à Medicina e Biologia.

Aprovado em:

Banca Examinadora

Prof. Dr.

Instituição:

Assinatura:

Prof. Dr.

Instituição:

Assinatura:

Prof. Dr.

Instituição: Assinatura: 


\section{DEDICATÓRIA}

Ao meu marido, William; à minha mãe Isabel e às minhas filhas Ayla, alegria do meu viver, e Ariadne, a qual espero sua chegada. 


\section{AGRADECIMENTOS}

Agradeço a Deus por ter me dado forças para realizar meu trabalho e conseguir completar esta etapa da minha vida acadêmica.

Ao meu marido, que sempre esteve ao meu lado, me ajudou incondicionalmente, e que sem ele eu não sobreviveria.

À minha filha, Ayla, que sempre me esperou até muito tarde, enquanto eu estava estudando.

À minha mãe, que abdicou de sua vida em outra cidade para poder me dar apoio em meus estudos.

Ao meu orientador, Prof. Dr. Alessandro Martins da Costa, que sempre teve paciência em esclarecer muito detalhadamente minhas dúvidas.

Aos funcionários Galo, PP, Tião, entre outros, da Sociedade Portuguesa de Benificência Hospital Imaculada Conceição, de Ribeirão Preto, que me ajudaram e me orientaram nessa jornada.

Ao Dr. Eduardo Andery Abbud, que disponibilizou o Serviço de Radiodiagnóstico do Hospital.

À Dra. Maristela Carbol Patta, que me ajudou num momento de muita dificuldade, e sempre que precisei ela estava pronta para me atender.

À minha família eterna, por sempre acreditar em mim.

À CAPES (Coordenação de Aperfeiçoamento de Pessoal de Nível Superior), pela concessão da bolsa de mestrado.

À Universidade de São Paulo (USP), pela infra-estrutura oferecida. 
"Um sonho sonhado sozinho é um sonho. Um sonho sonhado junto é realidade".

Raul Seixas 


\section{Resumo}

Os receptores de imagem radiográfica digitais estão gradualmente substituindo os sistemas tela-filme em radiodiagnóstico. Atualmente a radiografia computadorizada (CR - Computed Radiography) é a modalidade digital mais comum em muitos serviços de radiodiagnóstico. É baseada na utilização de fósforos foto estimuláveis, que também são conhecidos como fósforos de armazenamento. Quando sistemas tela-filme são utilizados como receptores de imagem, uma dose de radiação inadvertidamente alta no paciente irá resultar em um filme escuro, que fornece feedback imediato para o técnico em radiologia quanto aos fatores de técnica utilizados e a dose relativa. No entanto, quando receptores de imagem digitais são usados, uma dose alta no paciente pode produzir imagens excelentes o que resulta em uma tendência de se utilizar doses mais elevadas do que o necessário. A adequada justificação e otimização dos procedimentos exige o conhecimento dos valores típicos de dose em pacientes em um determinado serviço de radiodiagnóstico. O termo índice de exposição refere-se à dose absorvida na placa fósforo. Após a leitura da imagem com um sistema de laser o histograma dos sinais é computado e o índice de exposição é determinado com base nos valores dos pixels (picture elements) usando uma relação logarítmica. Os fabricantes dos sistemas de $C R$ oferecem os índices de exposição como um salvaguarda contra doses altas, mas a base para os valores recomendados não é clara. Além disso, os índices de exposição não estão diretamente relacionados com a dose no paciente. O resultado deste trabalho estabelece um método indireto para calcular os valores de dose de entrada, com base no índice de exposição, em pacientes submetidos a exames diagnósticos em sistemas de raios $\mathrm{X}$ com $C R$. Esses valores de dose de entrada obtidos foram então comparados com valores obtidos também indiretamente com base em avaliações de rendimento dos tubos de raios $\mathrm{X}$. 


\section{Abstract}

The receptors of digital radiography image are gradually substituting the screenfilm systems in radiodiagnostic. Currently, computed radiography is the most common method in many radiography services. It is based in the application of photostimulable phosphorus, that are also known as phosphorus storage. When screen-film systems are utilized as image receptors, an inadvertently high dose of radiation in the patient will result in a dark film screen, that inputs immediate feedback to the radiology technician as to the technical factors utilized and the relative dose. However, when digital image receptors are used, a high dose in the patient can produce excelent images which result in a tendency of using higher doses than necessary. The adequate extenuation and optimization of the procedures demand the knowledge of the characteristic dose values in patients in a determined radiodiagnostic service. The term exposure index refers to the absorbed dose in the phosphorus plate. Following the image reading with a laser system the histogram of signs is computed and the exposure index is determined in the basis of picture elements using a logarithmic relationship. The manufacturer of the systems of pixels offer the exposure indexes as a safeguard against high doses, but the basis for the recommended values are not clear. Moreover, the exposure indexes are not directly related to the dose in patients. The aim of this work was to establish an indirect method to estimate the values of entrance doses in patients who have undergone diagnostic exams in $\mathrm{X}$ ray systems with computed radiography based on exposure indexes. These values of entrance doses were compared with the values also obtained indirectly based in the measurements of the $X$ ray tubes output. 


\section{Lista de Figuras}

1 Gráfico mostrando como a Agfa define o índice de exposição $(\log M)$ e o nível de referência. . . . . . . . . . . . . . . . . . . . . . . . p.18

2 Geometria de irradiação para obter as curvas experimentais relacionando o kerma no ar incidente com o índice de exposição. Pode-se visualisar o tubo de raios $\mathrm{X}$, o objeto simulador geométrico de abdômen sobre a mesa de exame e a câmara de ionização no suporte em acrílico. p. 20

3 Kerma no ar incidente $\left(\mathrm{K}_{\mathrm{i}}\right)$ em função do Índice de Exposição (IE) para $80 \mathrm{kV}(\boldsymbol{\square}), 90 \mathrm{kV}(\bullet)$ e $100 \mathrm{kV}(\boldsymbol{\Delta})$ e objeto simulador geométrico de abdômen no aparelho de raios X convencional 1. . . . . . . . . . . . . p. 24

4 Kerma no ar incidente $\left(\mathrm{K}_{\mathrm{i}}\right)$ em função do Índice de Exposição (IE) para $80 \mathrm{kV}(\boldsymbol{\square}), 90 \mathrm{kV}(\bullet)$ e $100 \mathrm{kV}(\boldsymbol{\Lambda})$ e objeto simulador geométrico de abdômen no aparelho de raios X convencional 2 . . . . . . . . . . . . p. 25 


\section{Lista de Tabelas}

1 Parâmetros de ajuste $\mathrm{a}, \mathrm{b}$ e $\mathrm{c}$ de um polinômio de segundo grau relacionando o rendimento do tubo de raios $\mathrm{X}$ com a tensão para os aparelhos 1 e 2 (equação 2.3). . . . . . . . . . . . . . . . . . . . p. 23

2 Parâmetros de ajuste, $A$ e $B$, e coeficientes de correlação, $R^{2}$, obtidos com base nas determinações com o objeto simulador geométrico de abdômen exposto no aparelho de raios $X$ convencional $1 \ldots \ldots$. . . . p. 25

3 Parâmetros de ajuste, $A$ e $B$, e coeficientes de correlação, $R^{2}$, obtidos com base nas determinações com o objeto simulador geométrico de abdômen exposto no aparelho de raios $\mathrm{X}$ convencional 2 . . . . . . . p. 26

4 kerma no ar incidente, $\mathrm{K}_{\mathrm{i}}$, calculado com base na equação 2.2 e nos dados das tabelas 2 e 3, e também com base na equação 2.4, para 20 pacientes submetidos a exames diagnósticos de abdômen. 


\section{Sumário}

1 Introdução

p. 12

2 Materiais e métodos

p. 18

3 Resultados e discussões

p. 23

4 Conclusões

p. 29

Referências

p. 30 


\section{Introdução}

As fontes de radiação ionizante utilizadas em medicina proporcionam a maior contribuição à dose da população devido principalmente às fontes artificiais, sendo que acima de $90 \%$ desta contribuição provêm dos raios $X$ diagnósticos. Uma das razões para esta situação é o grande número de exames com raios $\mathrm{X}$ realizados a cada ano. O relatório do United Nations Scientific Committee on the Effects of Atomic Radiation (UNSCEAR) ${ }^{1}$ estima que o número anual de todos os tipos de exames médicos com raios $X$ no mundo foi de aproximadamente 2,1 bilhões em 2000, o que corresponde à frequência anual de 360 exames por 1000 habitantes. Esta frequência é aproximadamente 10\% maior que a estimativa prévia de 330 exames por 1000 habitantes para o período 1991-1995 ${ }^{2}$. Três quartos de todos os exames ocorrem nos países com nível de atenção à saúde I (um ou mais médicos por 1000 habitantes), os quais representam somente um quarto da população mundial. O Brasil, no relatório do UNSCEAR ${ }^{1}$, é classificado com o nível de atenção à saúde II (um médico por 10003000 habitantes), a partir de dados para o estado do Paraná (com uma população de 9 milhões de habitantes e um perfil social e econômico acima da média brasileira). Todos os dados sobre o Brasil que aparecem neste relatório são extrapolados a partir de dados para o estado do Paraná obtidos em um levantamento realizado em 1996. Apenas $1 \%$ de todos os exames ocorrem nos países com níveis de atenção à saúde menores (III e IV, um médico por 3000-10000 habitantes e um médico por mais que 10000 habitantes, respectivamente), que representam um quinto da população mundial. Contudo, é esperado um crescimento na radiologia médica nos países em desenvolvimento onde ainda faltam instalações e servicos de radiodiagnóstico. Portanto, há necessidade de se controlar estas doses e portanto otimizar o projeto e o uso de sistemas de raios $\mathrm{X}$ empregados em imagens médicas.

Um programa de garantia da qualidade em radiodiagnóstico pode levar à produção de radiografias de alta qualidade e exposição mínima do paciente ${ }^{3-6}$. A garantia da qualidade em radiodiagnóstico é constituída, entre outros, pelas avaliações da qua- 
lidade da imagem, pelas análises de rejeição de filme, pelas avaliações de dose em paciente e pelas medições dos parâmetros físicos dos vários componentes do sistema de raios $X$. Vários testes de controle da qualidade são necessários para garantir que os equipamentos de raios $\mathrm{X}$ estejam funcionando corretamente. Para dosimetria do paciente, o controle dos seguintes parâmetros é particularmente importante: tensão no tubo; camada semi-redutora (e filtração); dependência do rendimento do tubo com a tensão, tempo de exposição, corrente no tubo e produto corrente-tempo de exposição; reprodutibilidade do rendimento do tubo. As características desses parâmetros podem variar com o tempo, logo, os testes precisam ser feitos em intervalos regulares. Consequentemente é necessário entender como uma imagem é influenciada por esses parâmetros e como as suas características podem ser medidas utilizando-se de ferramentas apropriadas.

O Safety Series No. $115^{4}$ pede que sejam estabelecidos níveis de referência, isto é, valores de uma grandeza específica na prática de radiodiagnóstico, para exames típicos em grupos de pacientes típicos, para fornecer um guia sobre o que é alcançável nos procedimentos habituais quando são aplicadas as boas práticas correntes relativas ao radiodiagnóstico. Os níveis de referência devem ser derivados de dados obtidos em levantamentos em grande escala e modernizados com a evolução da tecnologia e das técnicas de radiodiagnóstico: esses níveis são baseados nos valores do terceiro quartil para as distribuições da grandeza específica encontradas nos levantamentos, isto é, $75 \%$ dos serviços de radiodiagnóstico estão abaixo destes valores de referência. A adoção do valor do terceiro quartil foi um enfoque puramente pragmático para ajudar a identificar os $25 \%$ dos serviços que necessitam mais urgentemente de um melhor controle da qualidade. Deve ser enfatizado que os níveis de referência de radiodiagnóstico não são limites regulamentados, mas devem ser vistos como um dispositivo prático para promover um melhor controle da qualidade local.

A publicação da International Commission on Radiation Units and Measurements (ICRU) sobre dosimetria de pacientes para raios $\mathrm{X}$ utilizados em imagens médicas ${ }^{7}$ divide as grandezas dosimétricas em básicas e de aplicação específica. As grandezas básicas são as grandezas fundamentais definidas no ICRU Report $60^{8}$. As grandezas de aplicação específica são grandezas dosimétricas práticas que são utilizadas para medições em radiodiagnóstico. 
A grandeza fundamental na qual os padrões nacionais são calibrados é o kerma (kinetic energy released per unit mass) no ar, $\mathrm{K}_{\mathrm{a}}$. A partir do kerma no ar pode-se determinar todas as demais grandezas de uso prático.

A grandeza kerma, $\mathrm{K}$, é o quociente de $\mathrm{dE}_{\mathrm{tr}}$ por $\mathrm{dm}$, em que $\mathrm{dE}_{\mathrm{tr}}$ é a soma da energia cinética inicial de todas as partículas carregadas liberadas pelas partículas não-carregadas (fótons e nêutrons) em uma massa dm de material, assim:

$$
K=\frac{d_{E_{t r}}}{d m} .
$$

A unidade para kerma é o $\mathrm{J} \cdot \mathrm{kg}^{-1}$ ou gray (Gy). A grandeza deve sempre ser definida com respeito ao material específico em que as interações estão ocorrendo, por exemplo kerma no ar.

No passado, para as medições em radiodiagnóstico, a grandeza exposição (unidade antiga: röentgen, R) era utilizada no lugar de kerma no ar. Valores de exposição em röentgen podem ser convertidos para kerma no ar em gray utilizando a conversão $0,876 \times 10^{-2} \mathrm{~Gy} / \mathrm{R}^{9}$.

A dosimetria de pacientes em radiodiagnóstico é complexa devido à variedade de técnicas para obtenção da imagem. Diversas grandezas de aplicação específica foram úteis no passado para dosimetria de pacientes em radiodiagnóstico. Entretanto, houve ambigüidade nos nomes e no uso destas grandezas. A ICRU ${ }^{7}$ fornece um conjunto consistente de grandezas, das quais, as grandezas kerma no ar incidente, kerma no ar na superfície de entrada e rendimento do tubo de raios $\mathrm{X}$, foram adotadas neste trabalho.

O kerma no ar incidente, $\mathrm{K}_{\mathrm{i}}$, é o kerma no ar a partir de um feixe incidente de raios $\mathrm{X}$ medido com o eixo do feixe central na posição da superfície do paciente ou objeto simulador. Apenas a radiação incidente sobre o paciente ou objeto simulador é medida, não incluindo a radiação retroespalhada.

A unidade para kerma no ar incidente é o $\mathrm{J} \cdot \mathrm{kg}^{-1}$ ou gray (Gy).

O kerma no ar na superfície de entrada, $\mathrm{K}_{\mathrm{e}}$, é o kerma no ar medido no eixo central do feixe na posição da superfície do paciente ou do objeto simulador. A radiação incidente no paciente ou no objeto simulador e a radiação retroespalhada estão incluídas.

A unidade para kerma no ar na superfície de entrada é o $\mathrm{J} \cdot \mathrm{kg}^{-1}$ ou gray (Gy). 
O kerma no ar na superfície de entrada está relacionado com o kerma no ar incidente pelo fator de retroespalhamento, FRE, assim:

$$
\mathrm{K}_{\mathrm{e}}=\mathrm{K}_{\mathrm{i}} \cdot \mathrm{FRE} \text {. }
$$

O rendimento do tubo de raios $X, R(d)$, é definido como o quociente do kerma ar, a uma distância especificada, d, a partir ponto focal do tubo de raios $\mathrm{X}$, pelo produto corrente no tubo-tempo de exposição, $\mathrm{P}_{\mathrm{lt}}$, assim:

$$
R(d)=\frac{K(d)}{P_{I t}}
$$

A unidade para o rendimento do tubo de raios $X$ é o $\mathrm{J} \cdot \mathrm{kg}^{-1} \cdot \mathrm{C}^{-1}$ ou $\mathrm{Gy} / \mathrm{C}$ ou Gy/A.s.

O principal objetivo da dosimetria de feixes de raios $X$ utilizados na produção de imagens médicas de pacientes é determinar grandezas dosimétricas para o estabelecimento e o uso de níveis de referência de radiodiagnóstico e para avaliação de risco comparativa ${ }^{10}$. Um objetivo adicional da dosimetria em radiodiagnóstico é a avaliação do desempenho do equipamento como parte de um programa de garantia da qualidade.

Em muitas situações, é interessante fazer-se avaliações para determinar as doses diretamente no paciente. Contudo, é preferível realizar essas avaliações usando um objeto simulador padrão para o controle dos parâmetros técnicos, comparação de diferentes sistemas e otimização. Várias técnicas de exame são utilizadas em radiodiagnóstico. Em alguns casos, dosímetros especializados são necessários, cujos projeto e desempenho devem corresponder às necessidades da determinação das doses clínicas. O uso de tais dosímetros e/ou a interpretação dos resultados obtidos pode requerer técnicas e conhecimentos especializados. É essencial padronizar os procedimentos para determinação da dose nas aplicações clínicas.

Avaliações de dose em radiodiagnóstico médico com objetos simuladores não podem fornecer uma estimativa direta da dose média para uma dada população de pacientes e não podem indicar as variações de dose vistas na prática por causa das diferenças no tamanho e na composição do paciente e na técnica radiográfica. Esta última inclui variações na qualificação dos técnicos que realizam os exames bem como 
as diferenças nos fatores de exposição que podem ser selecionados manualmente ou automaticamente.

Os receptores de imagem radiográfica digitais estão gradualmente substituindo os sistemas tela-filme em radiodiagnóstico ${ }^{11}$. Atualmente a radiografia computadorizada ( $C R$ - Computed Radiography) é a modalidade digital mais comum em muitos serviços de radiodiagnóstico. A $C R$ é baseada na utilização de fósforos foto estimuláveis, que também são conhecidos como fósforos de armazenamento. Os fósforos mais frequentemente usados são os da família dos haletos de bário flúor em forma de pó depositados sobre um substrato para formar uma placa ou tela. Os mecanismos de absorção de raios $X$ são idênticos aos das telas fosforescentes convencionais usadas em sistemas tela-filme. A diferença é que o sinal óptico não é derivado da luz emitida em resposta imediata à radiação incidente, mas sim de uma emissão posterior quando a imagem latente, que consiste em cargas armadilhadas, é oticamente estimulada e liberada das armadilhas metaestáveis. Isso desencadeia um processo chamado PSL (PhotoStimulated Luminescence - luminescência foto estimulada), resultante da emissão de luz de comprimento onda mais curto (azul) em quantidade proporcional à quantidade de raios $\mathrm{X}$ incidentes. $\mathrm{Na} C R$ a placa contendo o fósforo de armazenamento é colocada em um compartimento à prova de luz, exposta à imagem de raios $\mathrm{X}$ e então lida por varredura com um laser para liberar a PSL. A luz azul da PSL é coletada com um guia de luz e detectada com um PMT (PhotoMultiplier Tube - tubo fotomultiplicador). O sinal do PMT é digitalizado para formar a imagem ponto-a-ponto.

A imagem digital gerada é armazenada temporariamente em um disco rígido local. Muitos sistemas de $C R$ são ligados diretamente em impressoras laser que fazem cópias das imagens digitais em filmes. Os sistemas $C R$ muitas vezes servem também como pontos de entrada em um PACS (Picture Archiving and Communication System - Sistema de Comunicação e Arquivamento de Imagens) e, em tais casos, a imagem radiográfica digital é enviada para o PACS para interpretação pelo radiologista e arquivamento a longo prazo. A transmissão e armazenamento, na maior parte dos sistemas, é feita utilizando-se o padrão DICOM (Digital Imaging Communications in Medicine - comunicação de imagens digitais em medicina).

Quando sistemas tela-filme são utilizados como receptores de imagem, uma dose de radiação inadvertidamente alta no paciente irá resultar em um filme escuro, que fornece feedback imediato para o técnico em radiologia quanto aos fatores de técnica utilizados e a dose relativa. No entanto, quando receptores de imagem digitais são 
usados, uma dose alta no paciente pode produzir imagens excelentes o que resulta em uma tendência de se utilizar doses mais elevadas do que o necessário ${ }^{12}$.

A adequada justificação e otimização dos procedimentos exige o conhecimento dos valores típicos de dose em pacientes em um determinado serviço de radiodiagnóstico. No Brasil, a Portaria n. 453 do Ministério da Saúde, de 1 de junho de $1998^{5}$, exige que as doses nos pacientes sejam avaliadas como parte de um programa de garantia da qualidade em radiodiagnóstico. Comparações com os níveis de referência de radiodiagnóstico também são exigidas ${ }^{4,13-17}$.

Com a $C R$, não há nenhuma ligação física entre o receptor de imagem e o sistema gerador de raios $X$. Assim, não há possibilidade de capturar os fatores de técnica utilizados para um determinado paciente diretamente para o PACS. Os mais importantes fabricantes de sistemas de $C R$ têm tentado, para compensar esta dificuldade, a verificação dos fatores de técnica, introduzindo alguns índices de exposição relacionados com a luz emitida pelas placas de fósforo durante o processo de digitalização. $O$ termo índice de exposição refere-se à dose absorvida pela placa de fósforo. Após a leitura da imagem com o sistema laser o histograma dos sinais é computado e o índice de exposição é determinado com base nos valores dos pixels (picture elements) usando uma relação logarítmica ${ }^{18,19}$. Este parâmetro é armazenado no cabeçalho DICOM das imagens. Os fabricantes oferecem os índices de exposição como um salvaguarda contra doses altas, mas a base para os valores recomendados não é clara. Além disso, os índices de exposição não estão diretamente relacionados com a dose no paciente. Note-se que o índice de exposição é uma ferramenta que fornece indiretamente informações sobre a dose no paciente mas não é um substituto para a dose de entrada na pele (ou kerma no ar na superfície de entrada) ou para o produto dose área (ou produto kerma no ar área) ${ }^{7,20}$. Presentemente, um esforço para padronizar estes índices de exposição foi iniciado pela International Electrotechnical Commission ${ }^{21} \mathrm{e}$ pela American Association of Physicists in Medicine ${ }^{22}$ mas a aplicação prática ainda vai exigir vários anos.

O objetivo deste trabalho foi estabelecer um método indireto para calcular os valores de kerma no ar incidente, em pacientes submetidos a exames diagnósticos em sistemas de raios $X$ com $C R$, com base no índice de exposição extraído do cabeçalho DICOM das imagens. Os valores de kerma no ar incidente calculados foram comparados com valores obtidos também indiretamente com base em medições de rendimento dos tubos de raios $\mathrm{X}$, dos dados do paciente e dos fatores de técnica. 


\section{Materiais e métodos}

Este trabalho foi desenvolvido no serviço de radiodiagnóstico do hospital da Sociedade Portuguesa de Beneficência da cidade de Ribeirão Preto, no estado de São Paulo, Brasil.

Para a realização deste trabalho foi utilizado um digitalizador para $C R$ modelo CR85-X da Agfa. As imagens são processadas com o software MUlti Scale Imaging Contrast Amplification (MUSICA), também da Agfa. O índice de exposição, IE, também chamado de $\log \mathrm{M}$ pela Agfa, é calculado como a mediana dos valores de pixel logarítmicos no lobo principal do histograma da imagem (figura 1):

$$
\log M=2 \cdot S A L-3,9477,
$$

em que SAL é o nível médio do sinal (do inglês, Signal Average Level), proporcional aos valores de pixel.

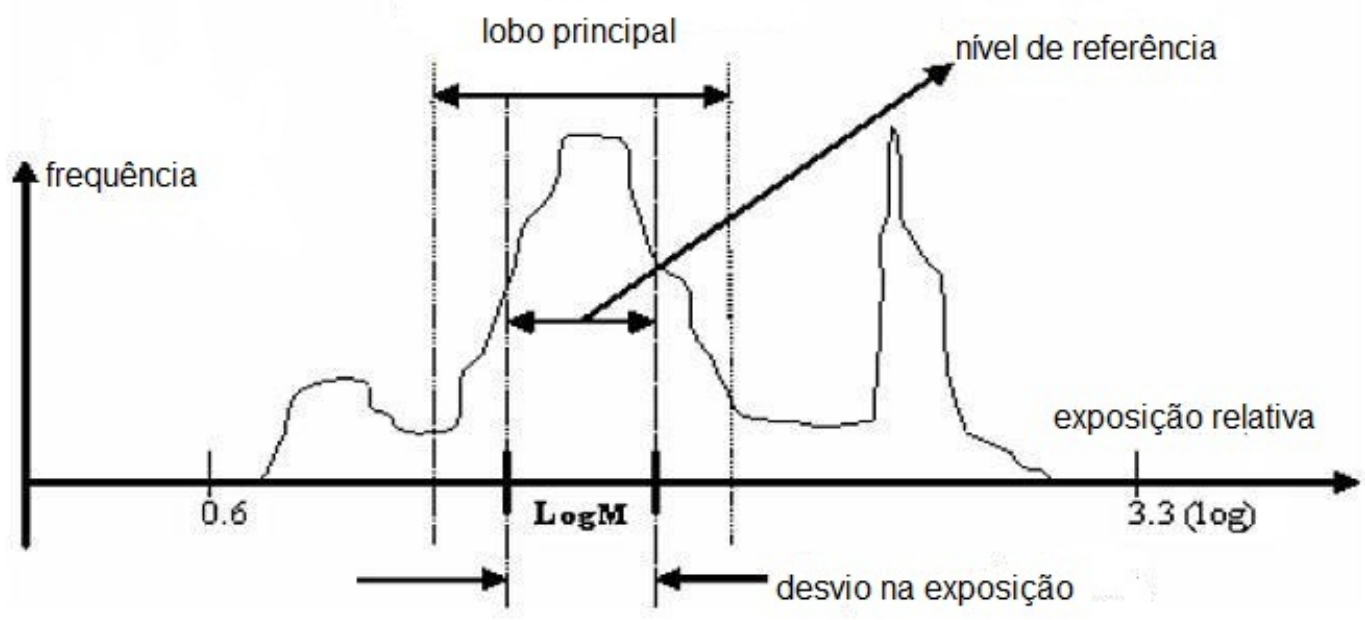

Figura 1: Gráfico mostrando como a Agfa define o índice de exposição $(\log M)$ e o nível de referência. 
Na figura 1, o pico da esquerda representa os valores mais baixos de nível de pixel, isto é, relacionados com a área colimada "escura" na imagem. O pico de direita representa as áreas "brilhantes" de exposição direta à radiação na imagem (valores elevados de nível de pixel). Ambos têm de ser removidos na avaliação do índice de exposição, uma vez que não estão relacionados com a área irradiada diretamente no paciente, que está de algum modo relacionado com o pico central na figura 1 . O IE é então avaliado usando a equação 2.1 e o seu valor é transferido para o cabeçalho DICOM da imagem. O nível de referência na figura 1 é o intervalo de valores considerados adequados, de acordo com o procedimento estabelecido pelo fabricante.

Devido à definição logarítmica de IE, um aumento de 0,3 significa uma duplicação da dose absorvida na placa de fósforo (o SAL é proporcional à dose e, portanto, $\Delta$ dose $\propto \Delta S A L \propto 10^{\Delta I E}=10^{0,3} \approx 2$ ).

Neste trabalho foram acompanhados 20 pacientes submetidos a exames diagnósticos de abdômen em sistemas de raios $\mathrm{X}$ com $C R$, registrando-se a espessura do paciente com o uso de um espessômetro, os fatores de técnica (tensão no tubo de raios $X$ e produto corrente-tempo de exposição), e o índice de exposição, obtido no processamento da imagem.

As curvas experimentais foram obtidas relacionando o kerma no ar incidente com o índice de exposição para diferentes tensões no tubo, utilizando um objeto simulador geométrico de abdômen.

Dois aparelhos de raios $X$ convencionais, denominados neste trabalho de 1 e 2 , da Shimadzu do Brasil, foram utilizados para obter o conjunto de curvas relacionando o kerma no ar incidente com o índice de exposição.

As tensões nos tubos utilizadas foram de $80 \mathrm{kV}, 90 \mathrm{kV}$ e $100 \mathrm{kV}$, que são as tensões mais utilizadas clinicamente no serviço de radiodiagnóstico nos exames de abdômen.

A geometria de irradiação para obter as curvas experimentais simula as condições clínicas, ou seja, $90 \mathrm{~cm}$ de distância fonte-receptor de imagem.

As determinações de kerma no ar incidente foram realizadas com uma câmara de ionização de placas paralelas PTW-Freiburg, modelo TN34069, acoplada a um eletrômetro digital PTW-Freiburg, modelo UNIDOS E T-10010. O conjunto câmara de ionização-eletrômetro foi devidamente calibrado. As medições de temperatura e pressão para a correção das leituras da câmaras de ionização foram obtidas utilizandose um altímetro/barômetro digital que também tem a função termômetro. Na função 
termômetro o intervalo de medição é de $-40^{\circ} \mathrm{C}$ até $+70^{\circ} \mathrm{C}$ e a resolução é de $0,1^{\circ} \mathrm{C}$. Na função barômetro o intervalo de medição é de $40 \mathrm{kPa}$ até $107 \mathrm{kPa}$ e a resolução é de $0,1 \mathrm{kPa}$.

Para o posicionamento da câmara de ionização no campo de radiação, para as medições no ar livre, foi utilizado um suporte em acrílico, fabricado na oficina mecânica do Departamento de Física da Faculdade de Filosofia, Ciências e Letras de Ribeirão Preto da Universidade de São Paulo,

A figura 2 mostra a geometria de irradiação para obter as curvas experimentais relacionando o kerma no ar incidente e o índice de exposição.

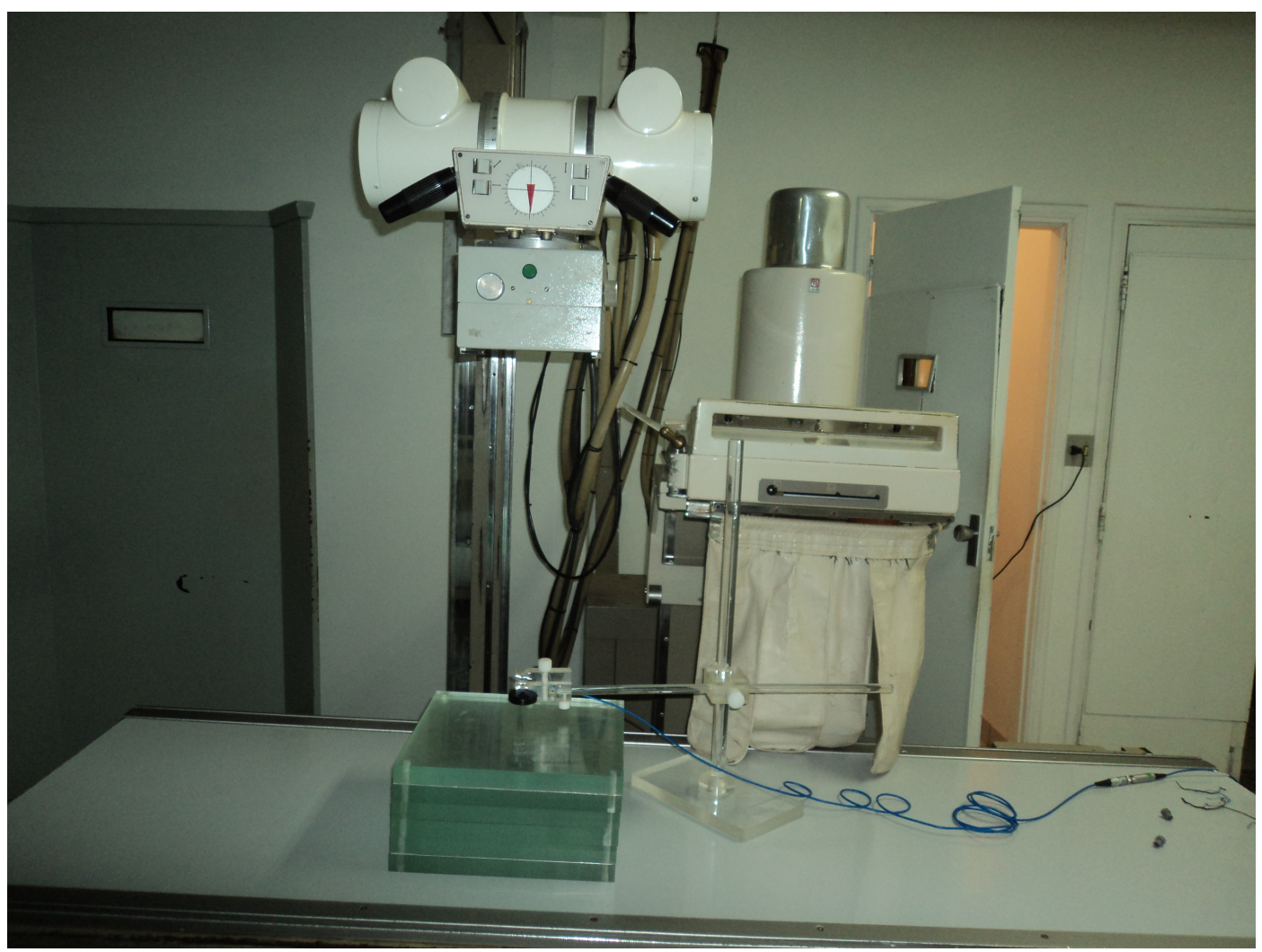

Figura 2: Geometria de irradiação para obter as curvas experimentais relacionando o kerma no ar incidente com o índice de exposição. Pode-se visualisar o tubo de raios $\mathrm{X}$, o objeto simulador geométrico de abdômen sobre a mesa de exame e a câmara de ionização no suporte em acrílico.

As curvas experimentais relacionando o kerma no ar incidente, $\mathrm{K}_{i}$, e o índice de exposição, IE, foram ajustadas a uma função exponencial:

$$
K_{i}=A \cdot \exp (B \cdot I E)
$$


em que A e B são os parâmetros de ajuste.

Assim, se o tipo de exame for abdômen, torna-se fácil a obtenção dos valores de kerma no ar incidente no paciente utilizando o índice de exposição e a equação 2.2. Uma explicação física da equação 2.2 pode ser obtida no apêndice $D$ do trabalho prévio de Vano e colaboradores ${ }^{23}$.

Baseando-se também no trabalho prévio de Vano e colaboradores ${ }^{23}$, estimou-se que a incerteza total no kerma no ar incidente obtido com base no índice de exposição foi de $50-100 \%$.

O kerma no ar incidente foi determinado, também de maneira indireta com base nas determinações do rendimento do tubo de raios $X$, no ar livre a uma determinada distância do foco utilizando-se a câmara de ionização, e levando-se em consideração a espessura do paciente e os fatores de técnica tensão no tubo, produto correntetempo de exposição e a distância foco-suporte do paciente. Esta metodologia para a dosimetria clínica em radiografia geral foi baseada no protocolo de dosimetria em radiodiagnóstico da International Atomic Energy Agency ${ }^{20}$ e nos trabalhos prévios de Tsapaki e colaboradores ${ }^{24}$ e Castañon e colaboradores ${ }^{25}$.

Para os dois aparelhos de raios $X$ convencionais utilizados, o rendimento do tubo de raios $X$ foi determinado para três valores diferentes de tensão na distância de $1 \mathrm{~m}$ do foco, e em seguida, foi ajustado um polinômio de segundo grau relacionando o rendimento, $\mathrm{R}$, e a tensão no tubo de raios $\mathrm{X}, \mathrm{kV}$ :

$$
\mathrm{R}(\mathrm{kV})=\mathrm{a} \cdot \mathrm{kV} \mathrm{V}^{2}+\mathrm{b} \cdot \mathrm{kV}+\mathrm{c}
$$

em que a, b e c são os parâmetros de ajuste.

Assim, foi calculado o kerma no ar incidente para cada paciente:

$$
K_{i}=R(k V) \cdot P_{I t} \cdot\left(\frac{1}{D F P}\right)^{2}
$$

em que $P_{\mathrm{It}}$ é o produto corrente-tempo de exposição e DFP é a distância-foco pele, obtida a partir da distância foco-suporte do paciente e da espessura do paciente.

Com base no rendimento do tubo de raios $X$, estimou-se que a incerteza total no kerma no ar incidente obtido foi de $25-30 \%$.

Antes da coleta dos dados foram realizados os testes de controle de qualidade nos aparelhos de raios $X^{26-28}$. Neste controle foram avaliados os parâmetros tensão 
no tubo, camada semi-redutora, linearidade do kerma no ar com o produto correntetempo de exposição e tempo de exposição, como também foram seguidos os procedimentos recomendados para equipamentos de raios $X$ diagnósticos convencionais ${ }^{26-28}$.

Para as medições de tensão no tubo de raios $X$, tempo de exposição e kerma no ar foi utilizado um medidor multipropósito PTW-Freiburg modelo Diavolt Multi. Para a determinação das camadas semi-redutoras foram utilizadas placas de alumínio puro (99\%) com espessuras de 0,5 mm (2 placas) e $1,0 \mathrm{~mm}$ (3 placas). 


\section{$3 \quad$ Resultados e discussões}

A exatidão da tensão no tubo de raios $X$ estava dentro de $\pm 9,6 \%$ para os dois aparelhos na faixa de $80-120 \mathrm{kV}$, e a reprodutibilidade foi melhor que $5,4 \%$. Enquanto que, a exatidão deve estar dentro de $\pm 10 \%$ e a reprodutibilidade deve ser melhor que $10 \%{ }^{27}$.

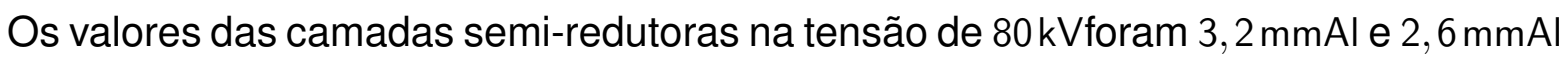
para os aparelhos de raios $X 1$ e 2 , respectivamente. O valor mínimo de camada semiredutora para uma tensão no tubo raios $X$ de $80 \mathrm{kV}$ deve ser 2, $6 \mathrm{mmAl}$ para um aparelho trifásico ${ }^{27}$, como é o caso dos dois aparelhos de raios $X$ convencionais utilizados neste trabalho.

A reprodutibilidade do kerma no ar, para a tensão de $80 \mathrm{kV}$ para os dois aparelhos, foi melhor que $6,1 \%$ em todos os casos, e a linearidade do kerma no ar com o produto corrente-tempo de exposição foi 3,7\% para o aparelho 1 e para o aparelho 2 foi 2,4\%. A reprodutibilidade deve ser melhor que $10 \%$ e a linearidade deve melhor que $20 \%{ }^{27}$.

A exatidão do tempo de exposição estava dentro de $\pm 15 \%$ para o aparelho 1 e de $\pm 25 \%$ para o aparelho 2 , e a reprodutibilidade foi melhor que $1,5 \%$ para o aparelho $1 \mathrm{e}$ que $15 \%$ para o aparelho 2 . A exatidão deve estar dentro de $\pm 10 \%$ e a reprodutibilidade deve ser melhor que $10 \%{ }^{27}$.

A tabela 1 mostra os parâmetros de ajuste de um polinômio de segundo grau relacionando o rendimento do tubo de raios $X$ com a tensão para os aparelhos 1 e 2 (equação 2.3.

Tabela 1: Parâmetros de ajuste a, b e c de um polinômio de segundo grau relacionando o rendimento do tubo de raios $X$ com a tensão para os aparelhos 1 e 2 (equação 2.3).

\begin{tabular}{cccc}
\hline Aparelho & $\mathrm{a}$ & $\mathrm{b}$ & $\mathrm{c}$ \\
\hline 1 & $-0,0000127648$ & 0,00397 & $-0,19418$ \\
2 & 0,0000549506 & $-0,0096$ & 0,42592 \\
\hline
\end{tabular}


As figuras 3 e 4 mostram o kerma no ar incidente em função do índice de exposição para $80 \mathrm{kV}, 90 \mathrm{kV}$ e $100 \mathrm{kV}$, e objeto simulador geométrico de abdômen para os aparelhos de raios $X$ convencionais 1 e 2 , respctivamente.

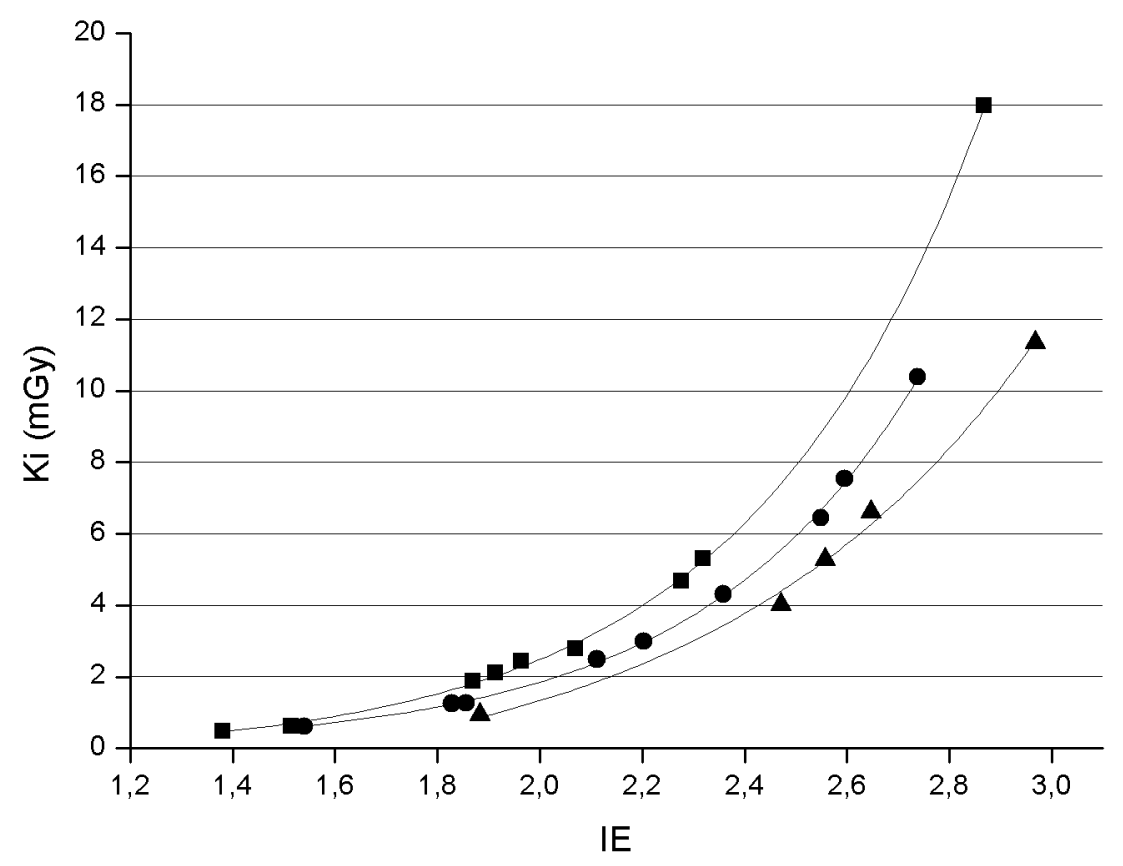

Figura 3: Kerma no ar incidente $\left(\mathrm{K}_{\mathrm{i}}\right)$ em função do Índice de Exposição (IE) para $80 \mathrm{kV}(\boldsymbol{\square}), 90 \mathrm{kV}(\bullet)$ e $100 \mathrm{kV}(\boldsymbol{\Delta})$ e objeto simulador geométrico de abdômen no aparelho de raios $X$ convencional 1 . 


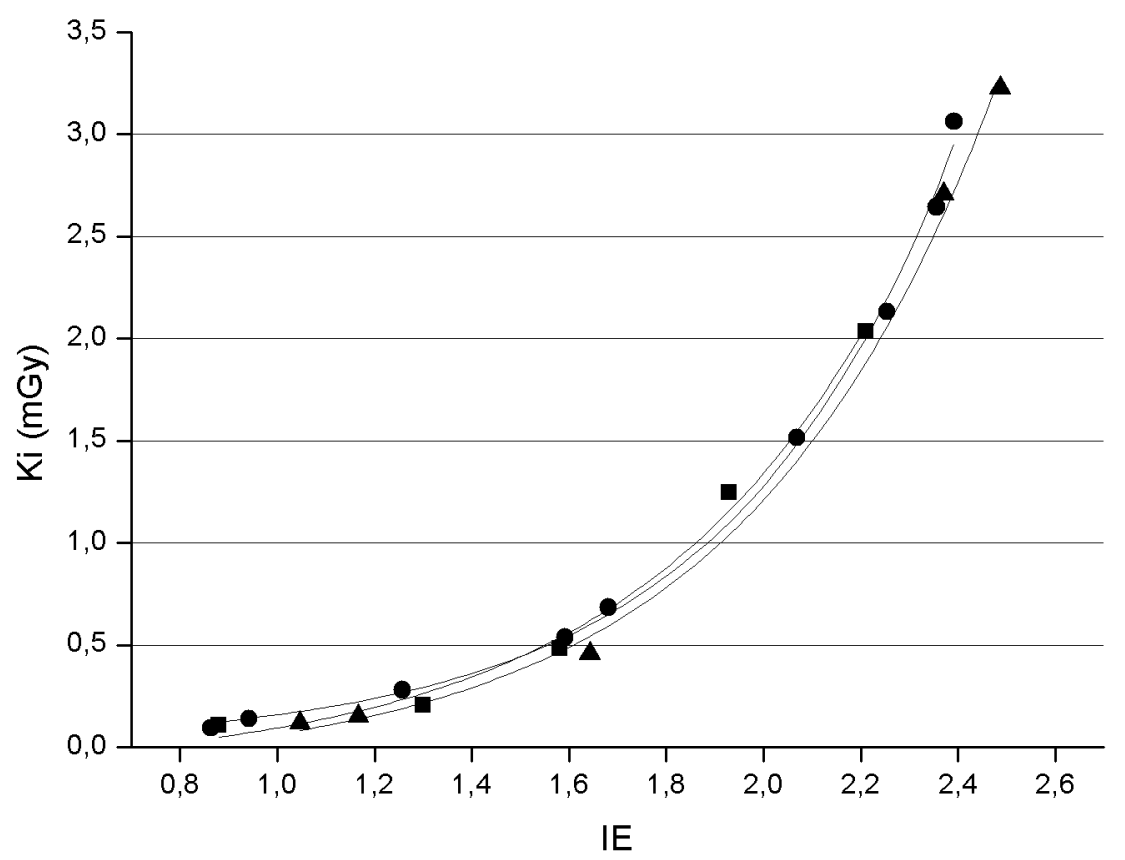

Figura 4: Kerma no ar incidente $\left(\mathrm{K}_{\mathrm{i}}\right)$ em função do Índice de Exposição (IE) para $80 \mathrm{kV}(\boldsymbol{\square}), 90 \mathrm{kV}(\bullet)$ e $100 \mathrm{kV}(\boldsymbol{\Delta})$ e objeto simulador geométrico de abdômen no aparelho de raios $X$ convencional 2 .

As tabelas 2 e 3 mostram os parâmetros de ajuste e coeficientes de correlação obtidos com base nas medições no objeto simulador geométrico de abdômen nos aparelhos de raios $X$ convencionais 1 e 2 , respectivamente.

Tabela 2: Parâmetros de ajuste, A e B, e coeficientes de correlação, $R^{2}$, obtidos com base nas determinações com o objeto simulador geométrico de abdômen exposto no aparelho de raios $X$ convencional 1 .

\begin{tabular}{cccc}
\hline Potencial no tubo $(\mathrm{kV})$ & $\mathrm{A}(\mathrm{mGy})$ & $\mathrm{B}$ & $\mathrm{R}^{2}$ \\
\hline 80 & 0,0264 & 2,274 & 0,99937 \\
90 & 0,0173 & 2,337 & 0,99902 \\
100 & 0,033 & 1,97 & 0,98735 \\
\hline
\end{tabular}


Tabela 3: Parâmetros de ajuste, A e B, e coeficientes de correlação, $R^{2}$, obtidos com base nas determinações com o objeto simulador geométrico de abdômen exposto no aparelho de raios $\mathrm{X}$ convencional 2 .

\begin{tabular}{cccc}
\hline Potencial no tubo $(\mathrm{kV})$ & $\mathrm{A}(\mathrm{mG})$ & $\mathrm{B}$ & $\mathrm{R}^{2}$ \\
\hline 80 & 0,0168 & 2,179 & 0,98787 \\
90 & 0,0216 & 2,056 & 0,99057 \\
100 & 0,014 & 2,19 & 0,99545 \\
\hline
\end{tabular}

A tabela 4 mostra o kerma no ar incidente calculado com base na equação 2.2 e nos dados das tabelas 2 e 3, e também com base na equação 2.4, para 20 pacientes submetidos a exames diagnósticos de abdômen, sendo que 10 deles foram no aparelho 1 e os outros 10 no aparelho 2 . 
Tabela 4: kerma no ar incidente, $\mathrm{K}_{\mathrm{i}}$, calculado com base na equação 2.2 e nos dados das tabelas 2 e 3, e também com base na equação 2.4, para 20 pacientes submetidos a exames diagnósticos de abdômen.

\begin{tabular}{|c|c|c|c|}
\hline Pacientes & $\mathrm{K}_{\mathrm{i}}$ (mGy), eq. 2.2 & $\mathrm{~K}_{\mathrm{i}}$ (mGy), eq. 2.4 & Desvio absoluto (mGy) \\
\hline 1 & 2,364 & 2,347 & 0,017 \\
\hline 2 & 0,941 & 0,972 & 0,030 \\
\hline 3 & 0,909 & 0,939 & 0,030 \\
\hline 4 & 0,890 & 0,939 & 0,049 \\
\hline 5 & 0,889 & 0,939 & 0,051 \\
\hline 6 & 0,879 & 0,939 & 0,061 \\
\hline 7 & 0,873 & 0,939 & 0,066 \\
\hline 8 & 0,871 & 0,939 & 0,069 \\
\hline 9 & 2,003 & 2,117 & 0,113 \\
\hline 10 & 2,147 & 2,347 & 0,199 \\
\hline 11 & 2,485 & 2,266 & 0,219 \\
\hline 12 & 0,488 & 0,939 & 0,451 \\
\hline 13 & 0,488 & 1,203 & 0,715 \\
\hline 14 & 2,523 & 3,911 & 1,388 \\
\hline 15 & 2,483 & 4,053 & 1,570 \\
\hline 16 & 0,719 & 2,396 & 1,677 \\
\hline 17 & 2,122 & 4,239 & 2,117 \\
\hline 18 & 2,075 & 4,239 & 2,164 \\
\hline 19 & 1,721 & 3,929 & 2,208 \\
\hline 20 & 1,725 & 4,080 & 2,354 \\
\hline Média & 1,480 & 2,234 & \\
\hline Desvio padrão & 0,742 & 1,355 & \\
\hline 3o. Quartil & 2,141 & 3,925 & \\
\hline
\end{tabular}

Levando-se em consideração as incertezas estimadas nas determinações, os resultados indicam que o kerma no ar incidente determinado com base no índice de exposição leva ao mesmo valor determinado com base no rendimento do tubo de raios $X$. Contudo, a reprodutibilidade das determinações com base no índice de exposição é melhor. $O$ desvio padrão mostrado na tabela 4 para as determinações com base no índice de exposição implicitamente leva em consideração a incerteza devido a pequenas variações na espessura do paciente. $O$ desvio padrão mostrado na tabela 4 para 
as determinações com base no rendimento do tubo de raios $X$ leva em consideração a incerteza devido a não conformidade do tempo de exposição nominal com o tempo de exposição medido.

Com o conhecimento das camadas semi-redutoras nas tensões no tubo de raios $\mathrm{X}$ utilizados e dos tamanhos do campo de radiação na superfície do paciente, é possível obter-se o fator de retrosespalhamento ${ }^{29}$ e calcular o kerma no ar na superfície de entrada ou dose de entrada na pele, como é denominado o kerma no ar na superfície de entrada na legislação nacional ${ }^{5,27}$. 


\section{Conclusões}

Com os resultados deste trabalho pode-se concluir que:

- com base no índice de exposição extraído manualmente, no momento do processamento da imagem, foi estabelecido um método indireto para calcular os valores de kerma no ar incidente em pacientes submetidos a exames diagnósticos de abdômen em sistemas de raios $\mathrm{X}$ com $C R$;

- a automatização da extração de informações do cabeçalho DICOM das imagens pode possibilitar o gerenciamento de uma amostra grande de dados de dose em pacientes ${ }^{30}$ com possibilidades de aplicação do método para outros tipos de exames;

- os resultados destas informações poderão servir de base para a justificação e otimização dos procedimentos no serviço de radiodiagnóstico;

- os valores de kerma no ar incidente obtidos pelo método indireto estabelecido neste trabalho foram comparados com valores obtidos também indiretamente com base em avaliações de rendimento dos tubos de raios $\mathrm{X}$, dados do paciente e dos fatores de técnica;

- os resultados encontrados com as duas metodologias mostraram-se semelhantes quando levadas em consideração as incertezas estimadas nas determinações dos valores de kerma no ar, embora a reprodutibilidade das determinações com base no índice de exposição seja melhor.

- a vantagem de se utilizar o índice de exposição é que não há a necessidade do conhecimento dos dados do paciente e dos fatores de técnica, uma vez que o kerma no ar incidente esta relacionado diretamente com o índice de exposição. 


\section{Referências}

1 UNITED NATIONS. Sources and Effects of lonizing Radiation: United Nations Scientific Committee on the Effects of Atomic Radiation (UNSCEAR) 2000 Report to the General Assembly with Scientific Annexes Vols.I \& II. New York, NY: UN, 2000.

2 UNITED NATIONS. Sources and Effects of lonizing Radiation: United Nations Scientific Committee on the Effects of Atomic Radiation (UNSCEAR) 1996 Report to the general assembly with scientific annexes. New York, NY: UN, 1996.

3 WORLD HEALTH ORGANIZATION. Quality assurance in diagnostic radiology. Geneva: WHO, 1982.

4 FOOD AND AGRICULTURE ORGANIZATION OF THE UNITED NATIONS; INTERNATIONAL ATOMIC ENERGY AGENCY; INTERNATIONAL LABOUR ORGANISATION; NUCLEAR ENERGY AGENCY OF THE ORGANISATION FOR ECONOMIC CO-OPERATION AND DEVELOPMENT; PAN AMERICAN HEALTH ORGANIZATION; WORLD HEALTH ORGANIZATION. International Basic Safety Standards for Protection Against lonizing Radiation and for the Safety of Radiation Sources, Safety Series No. 115. Vienna: IAEA, 1996.

5 BRASIL. Ministério da Saúde. Secretaria de Vigilância Sanitária. Diretrizes de proteção radiológica em radiodiagnóstico médico e odontológico, Portaria n. 453, 1 de junho de 1998. Diário Oficial da União, Poder Executivo, Brasília, 2 jun. 1998.

6 INTERNATIONAL ATOMIC ENERGY AGENCY; PAN AMERICAN HEALTH ORGANIZATION; WORLD HEALTH ORGANIZATION. Radiological protection for medical exposure to ionizing radiation, Safety Standards Series No. RS-G-1.5. Vienna: IAEA, 2002.

7 JOURNAL OF THE ICRU: ICRU Report 74, Patient dosimetry for $\mathrm{x}$ rays used in medical imaging. [S.I.]: Oxford University Press, v. 5, n. 2, 2005. 113 p.

8 INTERNATIONAL COMMISSION ON RADIATION UNITS AND MEASUREMENTS. ICRU Report 60, Fundamental quantities and units for ionizing radiation. Bethesda, MD: ICRU, 1998.

9 INTERNATIONAL COMMISSION ON RADIATION UNITS AND MEASUREMENTS. ICRU Report 47, Measurement of dose qquivalents from external photon and electron radiations. Bethesda, MD: ICRU, 1992.

10 ZOETELIEF, J.; PERNIČKA, F.; CARLSSON, G. A.; DANCE, D. R.; DEWERD, L. A.; DREXLER, G.; JÄRVINEN, H.; KRAMER, H. M.; NG, K. H. Dosimetry in diagnostic and interventional radiology: International Commission on Radiation Units and Measurements and IAEA activities. In: INTERNATIONAL SIMPOSYUM ON 
STANDARDS AND CODES OF PRACTICE IN MEDICAL RADIATION DOSIMETRY. Standards and codes of practice in medical radiation dosimetry. Proceedings of an International Symposium held in Vienna, Austria, 25-28 November 2002. Vienna: IAEA, 2003. v. 1, p. 387-404.

11 BUSHBERG, J. T.; SEIBERT, J. A.; LEIDHOLDT JR, E. M.; BOONE, J. M. The essential physics of medical imaging. 2. ed. Philadelphia, PA: Lippincott Willians \& Wilkins, 2002.

12 ANNALS OF THE ICRP: ICRP Publication 93, Managing patient dose in digital radiology. [S.I.]: Elsevier, v. 34, n. 1, 2004. 73 p.

13 ANNALS OF THE ICRP: ICRP Publication 60, 1990 Recommendations the International Radiological of Commission Protection,. [S.I.]: Elsevier, v. 21, n. 1-3, 1991. 201 p.

14 ANNALS OF THE ICRP: ICRP Publication 73, Radiological protection and safety in medicine. [S.I.]: Elsevier, v. 26, n. 2, 1996. 47 p.

15 GRAY, J. E.; ARCHER, B. R.; BUTLER, P. F.; HOBBS, B. B.; METTLER JR, F. A.; PIZZUTIELLO, R. J.; SCHUELER, B. A.; STRAUSS, K. J.; SULEIMAN, O. H.; YAFFE, M. J.; for the American Association of Physicists in Medicine Task Group on Reference Values for Diagnostic X-ray Examinations. Reference values for diagnostic radiology: application and impact. Radiology, v. 235, n. 2, p. 354-358, 2005.

16 ANNALS OF THE ICRP: ICRP Publication 103, The 2007 Recommendations of the International Commission on Radiological Protection. [S.I.]: Elsevier, v. 37, n. 2-4, 2007. 332 p.

17 ANNALS OF THE ICRP: ICRP Publication 105, Radiological protection in medicine. [S.I.]: Elsevier, v. 37, n. 6, 2007. 64 p.

18 HUDA, W.; RILL, L. N.; BRUNER, A. P. Relative speeds of Kodak computed radiography phosphors and screen-film systems. Medical Physics, v. 24, n. 10, p. 1621-1628, 1997.

19 AMERICAN ASSOCIATION OF PHYSICISTS IN MEDICINE. Report 93. Acceptance testing and quality control of photostimulable storage phosphor imaging systems. Madison, WI: Medical Physics Publishing, 2006.

20 INTERNATIONAL ATOMIC ENERGY AGENCY. Dosimetry in diagnostic radiology: an international code of practice, Technical Report Series No. 457. Vienna: IAEA, 2007.

21 INTERNATIONAL ELECTROTECHNICAL COMMISSION. Medical electrical equipment - Exposure index of digital X-ray imaging systems - Part 1: Definitions and requirements for general radiography, Standard IEC 62494-1. 1. ed. Geneva, 2008.

22 SHEPARD, S. J.; WANG, J.; FLYNN, M.; GINGOLD, E.; GOLDMAN, L.; KRUGH, K.; LEONG, D. L.; MAH, E.; OGDEN, K.; PECK, D.; SAMEI, E.; WANG, J.; WILLIS, C. E. An exposure indicator for digital radiography: AAPM Task Group 116 (Executive Summary). Medical Physics, v. 36, n. 7, p. 2898-2914, 2009. 
23 VAÑO, E.; MARTINEZ, D.; FERNANDEZ, J. M.; ORDIALES, J. M.; PRIETO, C.; FLORIANO, A.; TEN, J. I. Paediatric entrance doses from exposure index in computed radiography. Physics in Medicine and Biology, v. 53, n. 12, p. 3365-3380, 2008.

24 TSAPAKI, V.; TSALAFOUTAS, I. A.; CHINOFOTI, I.; KARAGEORGI, A.; CARINOU, E.; KAMENOPOULOU, V.; YAKOUMAKIS, E. N.; KOULENTIANOS, E. D. Radiation doses to patients undergoing standard radiographic examinations: a comparison between two methods. British Journal of Radiology, v. 80, n. 950, p. 107-112, 2007.

25 CASTAÑON, P. G.; LOPEZ, M. L. E.; BEDOYA, V. F.; LUNA, R. B.; MARTIN, G. R. A dose index as a tool to estimate paediatric patient doses in digital projection radiography. Radiation Protection Dosimetry, v. 149, n. 4, p. 417-423, 2012.

26 AMERICAN ASSOCIATION OF PHYSICISTS IN MEDICINE. Report 74. Quality control in diagnostic radiology. Madison, WI: Medical Physics Publishing, 2002.

27 BRASIL. Ministério da Saúde. Agência Nacional de Vigilância Sanitária. Radiodiagnóstico Médico: Segurança e Desempenho de Equipamentos. Brasília: ANVISA, 2005.

28 INSTITUTE OF PHYSICS AND ENGINEERING IN MEDICINE. Report 91. Recommended standards for the routine performance testing of diagnostic $x$-ray imaging systems. York: IPEM, 2005.

29 PETOUSSI-HENSS, N.; ZANKL, M.; DREXLER, G.; PANZER, W.; REGULLA, D. Calculation of backscatter factors for diagnostic radiology using Monte Carlo methods. Physics in Medicine and Biology, v. 43, n. 8, p. 2237-2250, 1998.

30 COSTA, C.; FERREIRA, C. C.; BASTIÃO, L.; RIBEIRO, L.; SILVA, A.; OLIVEIRA, J. Dicoogle - an Open Source Peer-to-Peer PACS. Journal of Digital Imaging, v. 24, n. 5, p. 848-856, 2011. 